\title{
LOCALISATION RARE DU MELANOME MUQUEUX
}

\author{
B. HAMMAMI, J. MOALLA, I. ACHOUR, S. KALLEL, A. CHAKROUN, \\ I. CHARFEDDINE, A. GHORBEL \\ SERVICE ORL ET CHIRURGIE CERVICO-FACIALE \\ CHU HABIB BOURGUIBA SFAX
}

\begin{abstract}
RESUME
But: Les mélanomes muqueux primitifs des voies aéro-digestives sont rares. La localisation nasopharyngée est exceptionnelle. Son diagnostic et sa prise en charge posent beaucoup de problèmes.

A travers une observation rare de mélanome primitif du nasopharynx, nous allons rappeler les difficultés diagnostiques et thérapeutiques de cette entité.

Observation : Nous rapportons le cas d'une patiente âgée de 15 ans qui a consulté pour des tuméfactions cervicales bilatérales avec obstruction nasale, otalgie droite et céphalées holocrâniennes. L'examen a objectivé une tumeur du nasopharynx étendue aux choanes, dont la biopsie a conclu à un mélanome malin. Le bilan d'extension n'a pas objectivé de métastases à distance. La patiente a eu un curage ganglionnaire bilatéral suivi de 6 cures de chimiothérapie, mais elle est décédée au bout de 9 mois, suite à une poursuite évolutive massive.

Conclusion : Le mélanome primitif du rhinopharynx est extrêmement rare et survient exceptionnellement chez l'enfant et l'adolescent. II est rarement suspecté cliniquement. Son diagnostic positif est histopathologique mais pose beaucoup de problèmes. Son traitement est non codifié. C'est une tumeur de mauvais pronostic vu son potentiel métastatique, et sa localisation anatomique inaccessible.
\end{abstract}

Mots clés : Mélanome, nasopharynx, immunohistochimie, pronostic

\section{SUMMARY}

Purpose: Mucosal melanoma of the upper respiratory tract is rare. Nasopharyngeal involvement is extremely rare. The diagnosis and management of this tumour still remain difficult.

We present a new case of mucosal nasopharyngeal melanoma, to discuss diagnosis and therapeutic modalities.

Observation: A 15-year-old girl was referred to the department of otolaryngology because of bilateral cervical mass with bilateral nasal obstruction, right otalgia and chronic headache. Nasopharyngeal tumour was observed endoscopically. The histologic examination showed mucosal melanoma. No distant metastatic localisations were identified. The patient underwent cervical lymph node dissection followed by 6 courses of chemotherapy, but she died 9 months later because of loco-regional failures.

Conclusion: The primary malignant melanoma of nasopharyngeal is extremely rare disease particularly among child. Its histologic diagnosis is challenging. The treatment still remains controversial. The prognosis is poor because of anatomic localisation and high frequency of distant metastasis.

Key words: Melanoma, nasopharynx, immuno-histochemistry.

\section{INTRODUCTION}

Le mélanome malin primitif des voies aéro-digestives supérieures est rare. L'atteinte nasopharyngée est exceptionnelle et seul quelques cas ont été rapportés chez des patients jeunes. Le diagnostic est rarement évoqué cliniquement, et c'est l'examen histologique aidé par l'immunohistochimie qui permet de le confirmer. Le mélanome du nasopharynx est de très mauvais pronostic du fait de son haut potentiel métastatique. Le traitement pose beaucoup de problèmes et on dispose de peu de moyens efficaces dans cette localisation inaccessible chirurgicalement.

Nous rapportons une observation de mélanome malin primitif du nasopharynx parmi 5 cas de mélanome muqueux de la tête et du cou, afin de discuter les difficultés diagnostiques, et thérapeutiques, de cette localisation.

\section{OBSERVATIONS}

III s'agissait d'une patiente âgée de 15 ans, sans antécédents particuliers, qui nous a été adressée pour tuméfactions cervicales bilatérales évoluant depuis 1 mois associées à une obstruction nasale droite, otalgie droite et céphalées holocrâniennes le tout dans un contexte d'altération de l'état général.

L'examen endoscopique a trouvé une tumeur ulcérobourgeonnante rougeâtre comblant le nasopharynx envahissant les deux choanes.

L'examen cervical a objectivé un magma d'adénopathies jugulo-carotidien bilatéral, de $3 \mathrm{~cm}$ de grand axe à droite et de $2 \mathrm{~cm}$ à gauche. Ces adénopathies étaient fermes, indolores, fixées aux plans profond et superficiel avec une peau en regard saine.

L'otoscopie a montré un tympan d'aspect grisâtre et terne à droite. L'examen des nerfs crâniens était sans anomalies. 
Le bilan biologique, comportant une numération de la formule sanguine et un bilan inflammatoire, était normal. L'intradermoréaction à la tuberculine était à $7 \mathrm{~mm}$.

Le scanner cervico-facial a montré la présence d'un processus expansif comblant tout le cavum, étendu aux choanes, aux cavités nasales et à l'espace parapharyngé droit sans lyse osseuse. II a objectivé aussi de multiples adénopathies cervicales jugulo-carotidiennes bilatérales de taille variant entre 1 et $2 \mathrm{~cm}$ groupées en magmas à centre nécrosé (fig.1).
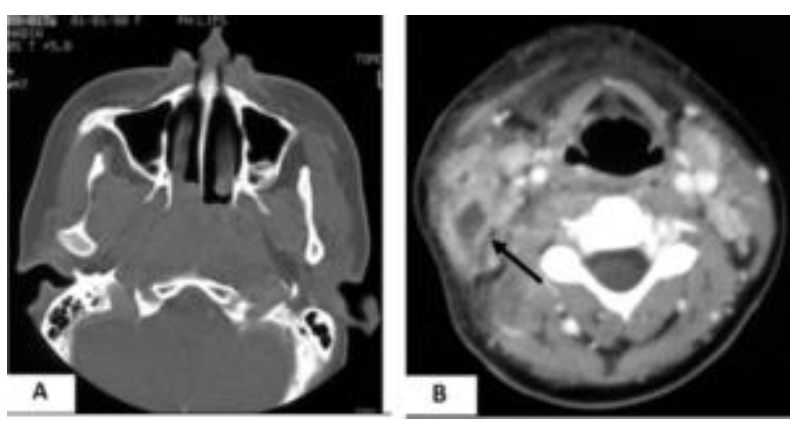

Fig. 1 : TDM en coupe axiale passant par le nasopharynx (A) et par la région cervicale (B) avec injection : Tumeur du nasopharynx étendue aux cavités nasales et à l'espace parapharyngé droit. Adénopathies cervicales nécrosées.

L'examen anatomopathologique avec étude immunohistochimique de la biopsie du cavum a montré une prolifération de cellules indifférenciés qui étaient fortement positives pour la vimentine, la protéine $\mathrm{S} 100$ (Ps 100) et faiblement positives pour le HMB45 et le Melan A (fig.2).

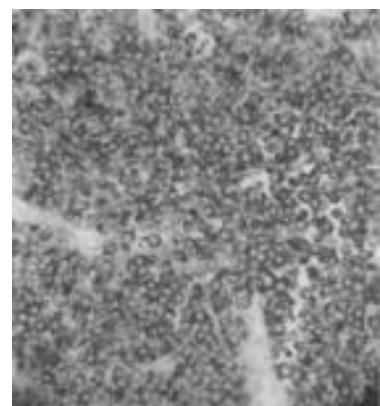

Fig.2 : Infiltration de la muqueuse nasopharyngée par des plages de cellules rondes à noyau macronucléolé exprimant la protéine $\mathrm{S} 100$

L'IRM du nasopharynx et cérébrale a trouvé que le mélanome était en isosignal T1, hypersignal T2, et prenait le produit de contraste de façon hétérogène, qu'il comblait le cavum, l'espace parapharyngé et qu'il s'étendait à la choane droite (fig.3). Ella a également permis d'éliminer une extension intracrânienne de la tumeur ainsi qu'une localisation méningée du mélanome.
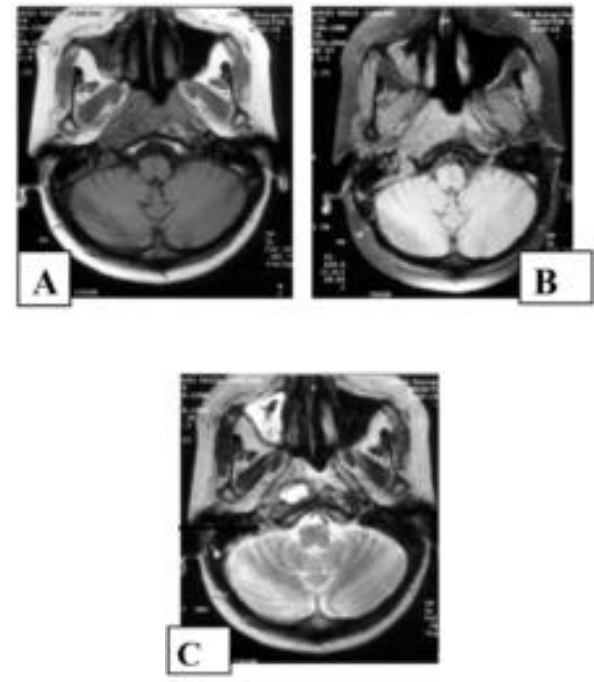

Fig.3 : IRM coupe axiale, séquence T1sans et avec injection de gadolinium (A et B) et T2 (C) : Tumeur du nasopharynx en iso T1 hyper T2 prenant le contraste de façon hétérogène.

Un examen dermatologique et ophtalmologique ont permis d'éliminer un mélanome cutané et oculaire. Un bilan d'extension, comportant une radiographie du thorax, une échographie abdominale, une scintigraphie osseuse et un scanner thoraco-abdominal était normal.

La tumeur a été classée mélanome malin du nasopharynx stade II.

La décision du comité multidisciplinaire était de faire un curage ganglionnaire bilatéral suivi d'une chimio-radiothérapie sur le nasopharynx et les aires ganglionnaires.

La patiente a eu un curage radical droit et fonctionnel gauche. Mais l'évolution était marquée par une poursuite évolutive ganglionnaire rapide et massive. Elle a reçu 6 cures de chimiothérapie, avec échec de contrôle locorégional. Elle est décédée au bout de 9 mois, par envahissement carotidien.

\section{DISCUSSION}

Les mélanomes muqueux de la tête et du cou sont des tumeurs rares et redoutables. Ils représentent 0,4 à $4 \%$ des cancers de cette région (1). Au niveau des voies aéro-digestives supérieures, ils se localisent préférentiellement au niveau de la cavité buccale et les cavités nasosinusiennes (2). La localisation nasopharyngée est exceptionnelle et ne représente que 0,01 à $0,05 \%$ des mélanomes muqueux de la tête et du cou $(3,4,5)$. Contrairement au mélanome cutané, son incidence ne semble pas en augmentation. Le mélanome muqueux nasopharyngé survient préférentiellement chez l'adulte de plus de cinquante ans $(3,6)$, avec une légère prédominance masculine. De très rares cas ont été rapportés chez des patients de moins de vingt ans. Quelques auteurs ont suggéré le rôle favorisant du tabac et l'exposition aux formaldéhydes (7). 
Il est très difficile d'évoquer le diagnostic de mélanome du nasopharynx cliniquement. En effet, cette tumeur rare chez des patients jeunes se manifeste par des signes rhinologiques non spécifiques. Les adénopathies à l'inverse du carcinome du nasopharynx, ne sont révélatrices que dans $16.6 \%$ des cas $(8,9)$. A l'examen endoscopique, la tumeur se présente comme une masse bourgeonnante souvent achromique (3).

Le diagnostic positif du mélanome malin du nasopharynx est exclusivement histologique et souvent difficile, nécessitant le recours à l'immunohistochimie (5). La tumeur est faite d'une prolifération de cellules très polymorphes, arrondies, globuleuses ou fusiformes. Les mitoses sont nombreuses et anormales. Cet aspect pose le problème de diagnostic différentiel avec les carcinomes indifférenciés, les lymphomes, les rhabdomyosarcomes et les neuroblastomes olfactifs. L'immunohistochimie permet de redresser le diagnostic en montrant des cellules exprimant la protéine S100 (PS100), la vimentine, HMB45, Melan A103, (3, 5, 10,11).

L'étude des limites tumorales et de l'extension locorégionale en particulier endocrânienne est basée sur I'IRM. La tumeur est souvent en hypo signal T1 et hyper signal T2, mais le signal peut être variable (11).

Le diagnostic de mélanome malin primitif du nasopharynx nécessite l'élimination de tout autre primitif par un examen dermatologique et ophtalmologique.

Les métastases à distance sont fréquentes (40 à 76\% des cas) et touchent les poumons, le foie, l'os et le cerveau (10). Une radiographie du thorax et une échographie abdominale sont réalisées systématiquement. Le scanner cérébral et la scintigraphie osseuse sont pratiqués uniquement devant des signes d'appels $(3,4)$.
En l'absence d'études randomisées et vu le nombre limité des malades dans les séries rétrospectives, la prise en charge des mélanomes nasopharyngés reste très controversée. Bien que le traitement des mélanomes muqueux se base, sur la chirurgie suivie d'une radiothérapie (10), la localisation profonde du nasopharynx, la découverte des tumeurs à des stades avancés, la nécessité de marges de sécurité suffisantes, rend cette chirurgie difficile, et souvent impossible.

La plupart des auteurs recommandent un curage bilatéral devant des adénopathies palpables $(3,4,5,6,10)$.

Les récidives locales précoces sont élevés chez les patients ayant reçu une radiothérapie exclusive $(5,9,10$, 11).

La radiothérapie post chirurgie semble donner un meilleur contrôle local et améliore la survie (4). Pour Wada, la radiothérapie post opératoire n'a pas donné d'effet sur les gros résidus tumoraux (12).

L'utilisation de la chimiothérapie pour les mélanomes muqueux à base de Déticène, Dacarbazine ou le Cis-platinum $(3,7,10,11)$ n'a prouvé son efficacité devant les mélanomes malins quelque soit sa localisation $(3,4)$.

Devant l'hypothèse d'une origine immunologique de ces tumeurs, certaines études sont en cours pour évaluer l'effet de l'interféron et l'interleukine2, dans le traitement de ces tumeurs (3).

Le contrôle local du mélanome du nasopharynx est difficile quelque soit le traitement. Le pronostic du mélanome primitif du nasopharynx est sombre avec une survie moyenne de 1 et 60 mois, selon le stade $(3,5,6,8,9,10$, 11).

\section{REFERENCES}

1. Grewal DS, Lele SY, Mallya SV, Baser B, Bahah NK, Rege JD. Malignant melanoma of the nasopharynx extending to the nose with metastasis in the neck. J Postgrad Med. 1994; 40:31-3.

2. Wening BM, Dulguerov P, Kapadia SB, Prasad ML, Fanfurg-Smith JC, Thompson LDR. Tumours of the nasal cavity and paranasal sinuses, neuroectodermal tumours. In Barnes L, Everson JW, Reichart P, Sidransky D, editors. WHO classification of tumours, pathology and genetics, head and neck tumours. Lyon: IARC Press; 2005:72-5.

3. Ronald J. P, Neil A.F and Jane L.M. Primary mucosal melanoma. J Am Acad Dermatol, 2007, 56 (5): 828-34.

4. Krengli. M, Masini. L, Kaanders. $J$ et al. Radiotherapy in the treatment of mucosal melanoma of the upper aerodigestive tract: Analysis of 74 cases. A Rare Cancer Network study. Int J Radiation Oncol Biol Physics, 2006; 65(3): 751-9.

5. Esteban S, Diaz C, Castano Z et al. Nasopharyngeal melanoma: a case report. An Otorrinolaringol Ibero Am, 1996; 23 (3) :285-9.

6. Chang A.E, Karnell L.H and Menck H.R. The national data base report on cutaneous and noncutaneous melanoma: a summary of 84,836 cases from the past decade. The American College of Surgeons commission on cancer and the
American Cancer Society. Cancer, 1998; 83: 1664-78.

7. Thompson L, Wieneke J A, Miettinen M. Sinonasal tract and nasopharyngeal malanomas. A clinicopathologic study of 115 cases with proposed staging system. The Am J Pathol 2003; 27:594-611.

8. Baltchford SJ, Koopmann CF, Coulthard SW. Mucosal melanoma of the head and neck. Laryngoscope 1986; 96: 929-34.

9. Kanellopoulos G, Longeaud-Desbrosses J, Meaux L, Kopiloff G, Paquelin F. Données récentes sur les mélanomes malins des muqueuses aéro-digestives supérieures à propos d'une observation. Ann Oto-Laryng 1984; 101 :553-9. 10. Enée V, Houliat T, Truilhé Y, Darrouzet V, Stoll D. Mélanomes malins des muqueuses naso-sinusiennes. Etude rétrospective à propos de 20 cas. Rev Laryngol Otol Rhinol 2000; $121: 243-50$.

11. Ramos R, Som PM, Solodnik P. Nasopharyngeal melanotic melanoma: MR characteristics. J Comput Assist Tomogr 1990; 14: 997-9.

12. Wada H., Nemoto K., Ogawa Y. et al. A multi-institutional retrospective analysis of external radiotherapy for mucosal melanoma of the head and neck in Northern Japan. Int J Radiation Oncol Biol Physics, 2004; 59(2): 495-500. 